\title{
Pulmonary Arteriovenous Fistula: Clinical and Histologic Spectrum of Four Cases
}

\author{
Soomin Ahn · Joungho Han \\ Hong Kwan Kim ${ }^{1}$. Tae Sung Kim² \\ Departments of Pathology and Translational \\ Genomics, ${ }^{\top}$ Thoracic Surgery, and ${ }^{2}$ Radiology, \\ Samsung Medical Center, Sungkyunkwan \\ University School of Medicine, Seoul, Korea \\ Received: January 6, 2016 \\ Revised: March 9, 2016 \\ Accepted: April 18, 2016 \\ Corresponding Author \\ Joungho Han, MD \\ Department of Pathology and Translational \\ genomics, Samsung Medical Center, \\ Sungkyunkwan University School of Medicine, \\ 81 Irwon-ro, Gangnam-gu, Seoul 06351, Korea \\ Tel: $+82-2-3410-2765$ \\ Fax: +82-2-3410-0025 \\ E-mail: haniho@skku.edu
}

\begin{abstract}
Pulmonary arteriovenous fistula (PAVF) is abnormally dilated vessels that provide a right-to-left shunt between pulmonary artery and pulmonary vein and is clinically divided into simple and complex type. Here, we report four cases of surgically resected sporadic PAVFs presenting various clinical and histologic spectrums. Cases 1 (a 57-old-female) and 2 (a 54-old-female) presented as incidentally identified single aneurysmal fistulas and the lesions were surgically removed without complication. On the other hand, case 3 (an 11-old-male) showed diffuse dilated vascular sacs involving both lungs and caused severe hemodynamic and pulmonary dysfunction. Embolization and surgical resection of the main lesion failed to relieve the symptoms. Case 4 (a 36-oldmale) had a localized multiloculated cyst clinically mimicking congenital cystic adenomatoid malformation. Microscopically, the lesion consisted of dilated thick vessels, consistent with the diagnosis of fistulous arteriovenous malformation/hemangioma.
\end{abstract}

Key Words: Lung; Arteriovenous fistula; Arteriovenous malformations
Pulmonary arteriovenous fistula (PAVF) in the lung is unusual. PAVF is described as abnormally dilated vessels that provide a right-to-left shunt between pulmonary artery and pulmonary vein. ${ }^{1,2}$ PAVFs are clinically and radiologically divided into simple and complex type and commonly associated with hereditary hemorrhagic telangiectasia (HHT). ${ }^{1}$ The treatment option of PAVFs varies depending on the location and severity of the lesion. Here we report four cases of surgically resected sporadic PAVF with different clinical and histologic presentations.

\section{CASE REPORTS}

\section{Case 1}

A 57-year-old woman visited the thoracic surgery department of the Samsung Medical Center, Seoul, for further evaluation of an abnormal finding on lung examination that had been incidentally detected by routine medical check-up. She denied any past medical history, and routine physical exams and laboratory tests were within normal limits. Chest computed tomography (CT) identified a single 4-mm-sized vascular dilation in the an- terior segment of left upper lobe (Fig. 1A). There was no abnormality in the rest of lung parenchyma. Under the impression of PAVF, video-assisted thoracoscopic surgery was performed. Cut section of the specimen revealed a 0.5 -cm-sized cystic lesion under the pleura. Microscopically, the lesion consisted of irregularly shaped aneurysmal dilation of thick vein (Fig. 1B). Additionally, elastic stain showed hypertrophic arteriopathy of pulmonary arterioles around the main aneurysmal vein (Fig. 1C). The patient was discharged without any post-operative complication.

\section{Case 2}

A 54-year-old woman presented with incidental abnormal finding on chest X-ray. She was on anti-hypertensive medicine due to hypertension. Her routine physical exams and laboratory tests were within normal limits. Chest CT revealed a 38-mmsized large aneurysmal sac in the right middle lobe (Fig. 1D). Due to the large size, right middle lobectomy was performed. Cut section of the specimen showed a thin-walled cyst with smooth whitish inner surface, measuring $38 \mathrm{~mm}$ in diameter 
(Fig. 1E). Elastic stain revealed the lesion to be consisted of aneurysmal dilation of both artery and vein (Fig. 1F). The patient was discharged without any postoperative complication.

\section{Case 3}

An 11-year-old boy visited emergency room due to hemoptysis. He was a known PAVF patient. He has had symptoms of cyanosis and clubbing of fingers since he was 4 years old and diagnosed with PAVF from chest CT work up. He had undergone coil embolization four times, which did not relieve the symptom. He showed general cyanosis and complained of dizziness and general weakness, low extremities in particular. His daily activity was limited and had difficulty in walking for long distance. $\mathrm{SpO}_{2}$ was checked as $70 \%$ on average. Despite several coil embolization attempts, cyanosis and signs of chronic heart failure worsened. Chest CT revealed severe vascular engorgement in both lungs (Fig. 1G). Right lower lobectomy was performed to remove the main part of the lesion. Cut section of the specimen showed many engorged vessels with blood clots mainly in the posterobasal segment, measuring approximately $9 \mathrm{~cm}$ in extent (Fig. 1H). Microscopically, many dilated veins filled with blood were identified (Fig. 1I). He was discharged with no specific postoperative complication. However, diffuse PAVFs still remained in other lobes. He constantly suffered from general weakness and $\mathrm{SpO}_{2}$ was still checked as $70 \%$ on average.

\section{Case 4}

A 36-year-old man was admitted to the thoracic surgery department for scheduled operation. The patient was treated with antibiotics due to lung abscess one month prior. He had had another episode of pneumonia in the right lung 20 years ago. Although his symptom was relieved, post-treatment chest CT still revealed a multi-cystic lesion in the lateral side of right lung showing plump vascular supply through intercostal arteries or pulmonary arteries (Fig. 1J). It showed no segmental or lobar distribution. Under the clinical impression of congenital cystic adenomatoid malformation (CCAM), surgical resection was planned. Right lower lobectomy with en bloc wedge resection of right upper lobe was performed. Cut section of the specimen revealed a multi-lobulated cystic lesion filled with blood, measuring $7 \mathrm{~cm}$ in extent (Fig. 1K). Rest of the pulmonary parenchyma showed no abnormality. Microscopic examination revealed that the cystic lining consisted of dilated thick vascular structure (Fig. 1L). Adjacent airway structures were also entrapped and dilated. The final pathologic diagnosis was given as fistulous arteriovenous malformation/hemangioma (AVMH).
The patient was discharged without any postoperative complication.

\section{DISCUSSION}

We describe four cases of surgically resected PAVFs exhibiting various clinical and histologic spectrum. PAVF is described as abnormally dilated vessels that provide a right-to-left shunt between pulmonary artery and pulmonary vein and is divided into simple and complex type., ${ }^{1,2}$ The literature review showed that abnormal communications between pulmonary arteries and veins have been given various names including PAVF, arteriovenous aneurysm, and arteriovenous hemangioma. ${ }^{1-3}$ While PAVFs have been often described as pulmonary arteriovenous malformation in several reports, ${ }^{1-5}$ we suggest that pulmonary AVMH should be used preferably to describe tumorous hemangioma lesion and PAVF should be used preferably to describe abnormal vascular communication. PAVFs are divided into simple and complex type, and the former is more common. ${ }^{1,5}$ Simple PAVFs are those of a well-defined peripheral nodule and may be rounded or multi-lobulated. ${ }^{1}$ Histologically, simple type presents as a single aneurysmal sac and contains a single segmental artery feeding the malformation. Complex PAVFs involve multiple abnormal vessels and consist of one or more lobulated venous sacs of variable size supplied by more than one feeding artery, often arising from adjacent segmental pulmonary artery branches. ${ }^{1}$ Complex PAVFs may involve whole lung segments or an entire lobe. ${ }^{1}$

Two patients (cases 1 and 2) presented with solitary aneurysmal vascular lesion, which corresponded to simple PAVF. These incidental lesions did not cause any clinical symptom and surgical removal was successfully performed. On the other hand, case 3 presented with diffuse vascular lesion causing arteriovenous shunt and was consistent with the diagnosis of complex PAVF. Complex PAVFs are supplied by more than one feeding artery. ${ }^{1}$ It caused serious respiratory problem and immobility in the patient 3. Lastly, case 4 showed a localized multi-lobular vascular lesion grossly mimicking pulmonary sequestration or CCAM. The histology of case 4 was unusual and also different from cases 1, 2, and 3 in that it had no definite aneurysmal sac or shunt formation. While AVMH is an acquired tumor-like condition characterized by complex thick-walled vessels, ${ }^{3}$ it was not certain whether case 4 was a tumorous condition of AVMH or not. However, we concluded that the terminology of AVMH would represent the histologic characteristics of case 4 the best in that there was definite localized overgrowth of thick vessels. Although it did not cause serious hemodynamic or pulmonary 

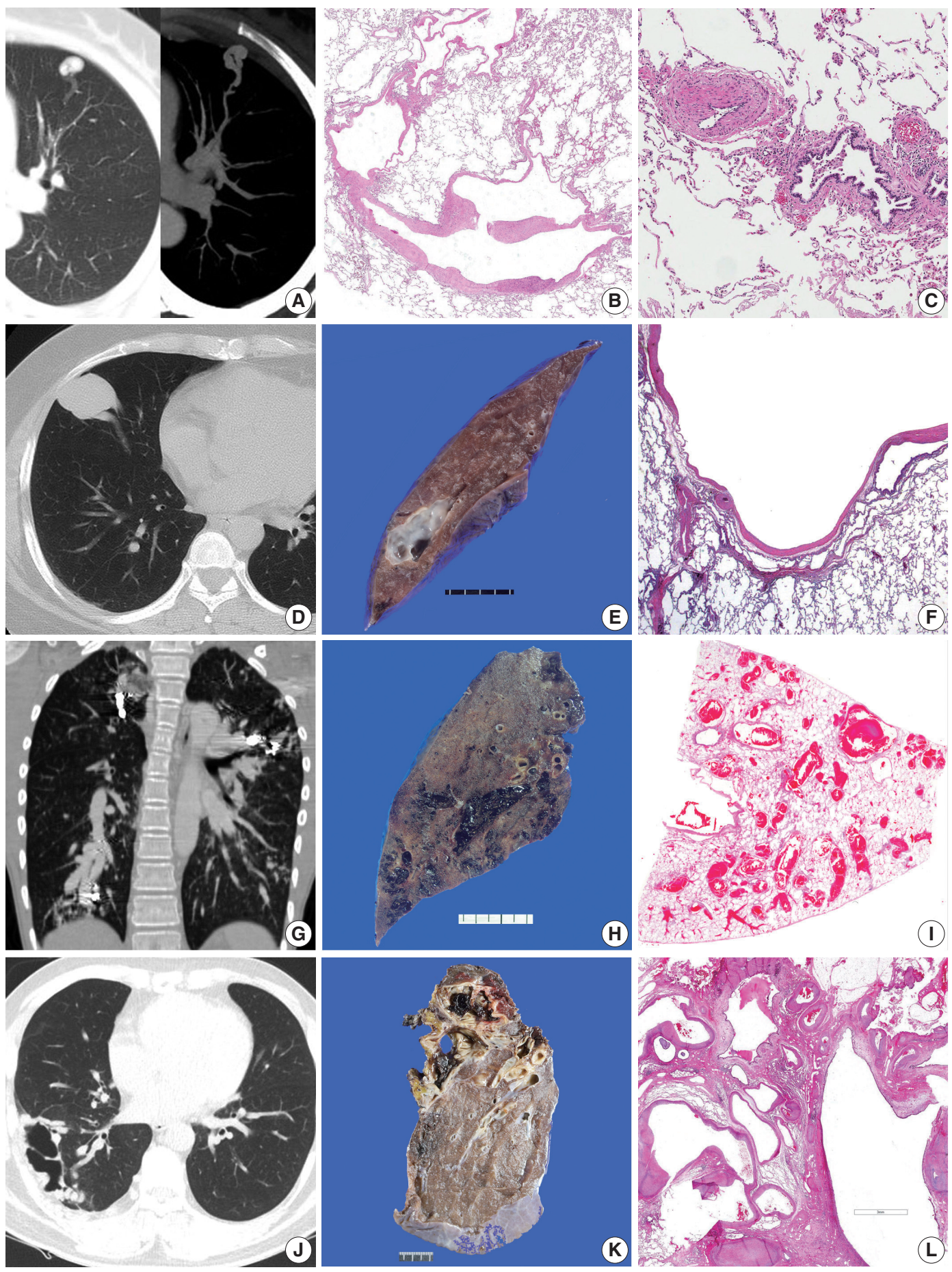

Fig. 1. Pulmonary arteriovenous fistula (PAVF). (A-F) Radiologic, gross, and microscopic features of two simple PAVF cases. (A, D) Both incidentally identified lesions are shown as dilated aneurysmal sacs in chest computer tomography (CT). (E) The cut section of case 2 shows a thin-walled cyst with smooth whitish inner surface. Microscopically, they consist of irregularly shaped aneurysmal dilation of thick vein (B, F) and hypertrophic arteriopathy of pulmonary arteriols around the main aneurysmal vein is accompanied in case 1 (C). (G-I) Radiologic, gross, and microscopic features of diffuse PAVF case. (G) Chest CT reveals severe vascular engorgement on both lungs. (H) On the cut section, many engorged vessels with blood clots are shown. (I) Microscopically, many dilated thin veins filled with blood are identified. (J-L) Radiologic, gross, and microscopic features of fistulous arteriovenous malformation/hemangioma. (J) Chest CT shows a multi-cystic lesion in the lateral side of right lung showing vascular supply through the intercostal arteries or pulmonary arteries. (K) The cut section of right lower lobectomy specimen shows a multi-lobulated cystic lesion filled with blood. (L) Microscopically, the cystic lining consists of dilated vascular structure. 
symptoms, his episodes of pneumonia may probably be related to this lesion.

While most PAVF patients are known to have HHT, patients in our series did not. PAVFs are not easily diagnosed routinely, due to its rarity and its unspecific findings on routine examinations. CT is generally considered the reference standard investigation for diagnosing PAVFs. ${ }^{1,4}$ In terms of treatment, embolization is considered first and surgical resection is performed for special indications. ${ }^{6,7}$ Therefore, it is rare for pathologists to encounter the histology of PAVFs. The four rare cases of surgically resected PAVFs described herein show various spectrums of clinical and histologic features. They can present as either localized or diffuse, and they can involve either thin or thick blood vessels.

\section{Conflicts of Interest}

No potential conflict of interest relevant to this article was reported.

\section{REFERENCES}

1. Gill SS, Roddie ME, Shovlin CL, Jackson JE. Pulmonary arteriove- nous malformations and their mimics. Clin Radiol 2015; 70: 96-110.

2. Gossage JR, Kanj G. Pulmonary arteriovenous malformations: a state of the art review. Am J Respir Crit Care Med 1998; 158: 643-61.

3. Ahn S, Jung S, Cho JH, Kim TS, Han J. A rare case of pulmonary arteriovenous hemangioma presenting as a peribronchial Mass. J Pathol Transl Med 2015 Nov 17 [Epub]. http://dx.doi.org/10.4132/ jptm.2015.10.15.

4. Nakayama M, Nawa T, Chonan T, et al. Prevalence of pulmonary arteriovenous malformations as estimated by low-dose thoracic CT screening. Intern Med 2012; 51: 1677-81.

5. White RI Jr, Mitchell SE, Barth KH, et al. Angioarchitecture of pulmonary arteriovenous malformations: an important consideration before embolotherapy. AJR Am J Roentgenol 1983; 140: 681-6.

6. Terry PB, White RI Jr, Barth KH, Kaufman SL, Mitchell SE. Pulmonary arteriovenous malformations. Physiologic observations and results of therapeutic balloon embolization. N Engl J Med 1983; 308: 1197-200.

7. Gupta P, Mordin C, Curtis J, Hughes JM, Shovlin CL, Jackson JE. Pulmonary arteriovenous malformations: effect of embolization on right-to-left shunt, hypoxemia, and exercise tolerance in 66 patients. AJR Am J Roentgenol 2002; 179: 347-55. 